OAI-PMH: http://www.indteca.com/ojs/index.php/Revista Scientific/oai

Artículo Original / Original Article

\title{
Propuesta de un Programa de Desarrollo del Departamento Metodológico Federación Deportiva del Cañar
}

Autor: Olger Estuardo Almache Tello Universidad Nacional de Educación, UNAE estuardo.almache@unae.edu.ec Azogues, Ecuador

\section{Resumen}

El presente artículo, propone un programa para el desarrollo del Departamento Técnico Metodológico de Federación Deportiva del Cañar basado en la metodología Matriz FODA (Fortalezas, Oportunidades, Debilidades y Amenazas), cuyo objetivo fue en cambiar las estructuras y funcionamiento del mismo planteando una propuesta de desarrollo. La investigación se realizó en los seis de los siete cantones que tiene la provincia del Cañar por ser éstos los que disponen de Ligas Deportivas Cantonales los cuales son: Azogues, Biblián, Cañar, El Tambo, Suscal y La Troncal. Para obtener información se aplicaron encuestas a 60 sujetos que conforman el cuerpo técnico (entrenadores, monitores, preparadores físicos), dirigentes deportivos y tres entrevistas a los directores de los departamentos técnicos del Ecuador; con una muestra intencionada de veinte y cuatro trabajadores mismos que permitieron confirmar la pertinencia del proyecto de investigación y su correspondiente propuesta metodológica.

Entre los principales hallazgos de esta investigación esta: que la estructura orgánica del Departamento Técnico Metodológico de la Federación Deportiva del Cañar no posee secciones de dirección metodológica lo que no le permite atender las necesidades reales de la provincia en el ámbito deportivo.

Palabras clave: técnico; metodología; investigación. 


\title{
Proposal of a Development Program of the Methodological Department of the Sports Federation of Cañar
}

\begin{abstract}
The present article proposes a program for the development of the Methodological Technical Department of the Cañar Sports Federation based on the SWOT Matrix methodology (Strengths, Opportunities, Weaknesses and Threats), whose objective was to change the structure and operation of the same, proposing a proposal of development. The investigation was carried out in the six of the seven cantons that the province of Cañar has, since these are the ones that have Cantonal Sports Leagues which are: Azogues, Biblián, Cañar, El Tambo, Suscal and La Troncal. To obtain information, surveys were applied to 60 subjects that make up the technical staff (coaches, monitors, physical trainers), sports leaders and three interviews to the directors of the technical departments of Ecuador; with an intentional sample of twenty-four workers that allowed to confirm the pertinence of the research project and its corresponding methodological proposal.

Among the main findings of this research is that the organizational structure of the Methodological Technical Department of the Sports Federation of Cañar does not have sections of methodological direction which does not allow it to meet the real needs of the province in the sports field.
\end{abstract}

Keywords: technical; methodology; research.

Date Received: 04-04-2018

Date Acceptance: 25-06-2018 


\section{Introducción}

El nivel de desarrollo del Deporte en el contexto mundial, es el resultado de la unión de varios aspectos, los mismos que organizado y fundamentado sobre bases científicas, técnicas y psicopedagógicas comprenden el factor de progreso más importante.

El deporte moderno no se concibe alejado del manejo y estudio de sus nuevas concepciones relacionadas con la planificación y estructuración del entrenamiento, esto junto a las ciencias aplicadas al deporte son motor impulsor de los grandes resultados de los atletas de esta era (Vasconcelos, 2000, pág. 26).

Una de las ciencias aplicadas al deporte de mayor importancia, es precisamente la Dirección Científica del Deporte, cuyos conceptos se aplican como herramienta que facilita el proceso de dirección, gestión, organización, planificación y evaluación deportiva en el mundo (Montero, 2006, pág. 57). En el contexto ecuatoriano, la actividad deportiva, no refleja los efectos de su aplicación en el campo social, peor aún, en el competitivo, los resultados demuestran el pobre desarrollo deportivo, lo que conlleva a establecer que el fenómeno social del deporte se encuentre estancado, es por eso la necesidad de emprender proyectos de investigación que ayuden a formular propuestas, soluciones y establecer estrategias de transformación (Forteza y Ranzolas, 1998, pág. 63).

Lo antes expuesto en esta introducción, se sustenta en la investigación de campo y las experiencias vividas durante su realización, y lo reflejado en los cuadros estadísticos y los gráficos respectivos, teniendo en consideración las 24 provincias existentes en el momento de la investigación, escogiendo el $32 \%$ de provincias del país como muestra. El objetivo planteado en esta investigación fue de manera general proponer un programa de desarrollo en el Departamento Técnico Metodológico aplicando las estrategias estructurales y de contenido en función de los técnicos y entrenadores deportivos de la 
Federación Deportiva del Cañar.

La naturaleza de la investigación considera al Departamento Técnico Metodológico de la Federación Deportiva del Cañar, como elemento de investigación prioritario, debido al hecho de ser la fuente organizativa y administrativa de la gestión deportiva que se desarrolla en su contexto, es así que en la provincia del Cañar no existe un programa para el desarrollo del Departamento Técnico Metodológico que permita el impulso exitoso del deporte de acuerdo con las características geográficas y económicas, lo que justifica nuestra investigación que está encaminada a tener un programa específico para el control del entrenamiento deportivo.

\section{La Dirección Técnico-Metodológica}

La Dirección Técnico Metodológica en el Ecuador ha tenido múltiples dificultades que en algún momento se han convertido en el freno del desarrollo del deporte de nuestro país, debido a la falta de una estructura organizada que apoye esta labor de manera técnica y científica, este fenómeno social también se refleja en algunos deportes en especial los de nivel profesional, en el caso del deporte amateur, la tendencia fundamental de los entrenadores es importar los sistemas y métodos de entrenamiento de países desarrollados deportivamente, hacia los deportistas en la búsqueda de medallas, olvidándose de la formación multilateral del deportista (Vinueza, 2004a, pág.17).

Un repaso a la situación problemática en el contexto de este documento establecería que uno de los factores fundamentales para que no existan logros deportivos en el Ecuador, está centrado en la falta de programas de enseñanza, que orienten al profesional de la Cultura Física y el Deporte a la consecución de objetivos (Vinueza, 2004b, pág. 23). La falta de una política de manejo deportivo, y la falta de profesionales especializados hace que la dirección de deporte no sea la más adecuada, en las Direcciones Técnico- 
Metodológicas Provinciales; lo que ha impedido crear una estructura sólida y sistemática que respete el desarrollo cronológico, psicológico del niño dentro de la Educación Física y Deporte.

En conclusión, el deporte debe funcionar como un sistema, donde todos los que trabajamos en él, contribuyamos a su desarrollo de forma organizada y planificada, debido que debemos entender que la parte fundamental del deporte es el deportista y quien lo forma es el entrenador, sin estos dos pilares que sustentan las instituciones deportivas, los demás componentes no existirían.

Investigando la incidencia de la Cultura Física en nuestro país; se puede revelar que es un punto muy álgido, que debería ser analizado con suma importancia. Las deficiencias más marcadas dentro de ésta y está basado en la falta de profesionales con experiencia en la rama de la Educación Física en los establecimientos educativos (escuelas), las clases de Cultura Física se basan solamente en el juego del Fútbol, pero casi sin ningún tipo de enseñanza de las técnicas elementales lo cual convierte la clase en un proceso empírico, no provechoso en el desarrollo de destrezas.

Considerando que el trabajo Técnico-Metodológico es un factor muy importante para direccionar el manejo del deporte en el Ecuador, vale la pena hacer una reseña histórica: En el año de 1985 en el Comité Olímpico Ecuatoriano crea el primer documento relacionado con esta actividad conocido como Plan de Desarrollo de la Cultura Física en el Ecuador, direccionado por el Director del Departamento Técnico Metodológico del COE de ese entonces; en 1994 la Federación Deportiva Nacional del Ecuador "FEDENADOR", empieza a impulsar el trabajo Técnico Metodológico a través de su Departamento Técnico Nacional y crea un plan emergente relacionado específicamente a la capacitación de las personas que en ese entonces podían cumplir esta labor en las Federaciones Deportivas Provinciales, en la actualidad, se ha logrado dar un paso muy importante, porque las 
Federaciones Deportivas Provinciales y los Organismos Superiores cuentan ya con un Departamento Técnico; lo que no se ha podido es, erradicar el empirismo, la improvisación y la desorganización del Deporte por la falta de dirección en el país (Vinueza, 2004c, pág. 38).

Es así que el Departamento Técnico Metodológico, llega a ser la estructura técnica más importante dentro de la dirección del deporte competitivo y responde directamente a los procesos pedagógicos de la enseñanza del deporte, sus tareas principales son: Planificar, organizar, orientar, controlar y evaluar el trabajo técnico-metodológico de los deportes y establecer los criterios científico-metodológicos para llevar adelante el proceso de selección, garantizar la capacitación científico-metodológica de los entrenadores, dosificar el número de sesiones semanales, cantidad de horas, así como los componentes de la carga de entrenamiento (Vinueza, 2004d, pág. 51).

Basado en el razonamiento dado en el párrafo anterior el Trabajo Técnico-Metodológico es el principal instrumento para llegar a los objetivos establecidos en la enseñanza deportiva. Irrespetando las tareas principales antes expuestas. El Departamento Técnico Metodológico en nuestro país, además de su responsabilidad establecidas en los orgánicos también cumplen otras funciones que tienen que ver con las actividades administrativas legislativas y gerenciales que impiden el normal desarrollo y cumplimiento de las funciones específicas de este Departamento. En los escaneos donde incide del trabajo técnico metodológico son:

1. Trabajo Técnico: Se orienta hacia la Planificación Deportiva, de forma que se propicien los resultados competitivos previstos.

2. Trabajo Metodológico: Se fundamenta en la calidad del proceso y la búsqueda de impulsar la Maestría Pedagógica de los entrenadores.

\section{Metodología}

La presente investigación se realizó en la Federación Deportiva del 
Cañar que está ubicada en el cantón Azogues capital de la provincia del Cañar que cuenta con una población de 206.981 habitantes. Políticamente cuenta con siete cantones: Azogues (capital provincial), La Troncal, Suscal, Cañar, El Tambo, Biblián y Déleg, mismos que constituyeron el universo de la investigación. Para conseguir esta ambiciosa meta por la expansión del universo se realizó una "Propuesta de un Programa de Desarrollo para el Departamento Técnico Metodológico de Federación Deportiva del Cañar" misma que consta de; un FODA de la institución, una nueva propuesta de la estructura orgánica funcional del Departamento Técnico Metodológico, un formato o guía para analizar y aprobar los documentos que el cuerpo técnico lo utiliza, estructuración de las visitas que se establece en la planificación (Visitas Técnicas, Visitas Metodológicas, Visita a los Cantones, Visitas a Competencia), reunión con el cuerpo técnico acordes a sus necesidades, formación del cuerpo multidisciplinario, un programa de evaluación al cuerpo técnico, plan de capacitación y masificación en la provincia.

Esta investigación, es un estudio de campo, ya que se desarrolló en las instalaciones de Ligas Deportivas Cantonales seleccionadas, de esta manera se obtuvo la información de los propios involucrados; es decir, entrenadores y monitores.

La población que sirvió de base para esta investigación fue de sesenta (60) entrenadores, monitores, preparadores físicos y tres (3) entrevistas a los directores de los departamentos técnicos del Ecuador; con una muestra intencionada de veinte y cuatro trabajadores mismos que permitieron confirmar la pertinencia del proyecto de investigación y su correspondiente propuesta metodológica.

Para el análisis de la información recopilada se hizo utilizando la estadística descriptiva. Los resultados obtenidos de esta investigación se interpretaron de acuerdo con una tabla de contrastación bosquejado en función de las variables existentes. 


\section{Análisis de Resultados de la Aplicación del Programa}

Como resultado del FODA aplicado se pudo obtener los datos que sirvieron como base para una nueva estructura del Departamento Técnico Metodológico (D.T.M.); dicha propuesta abajo descrita piensa mantener las líneas de dirección sujetas a este Departamento con la creación de secciones metodológicas acordes a la necesidad real de la provincia.

Diagrama № -1 . Propuesta de una Estructura del Departamento Técnico Metodológico de FEDECAÑAR (Federación Deportiva del Cañar).

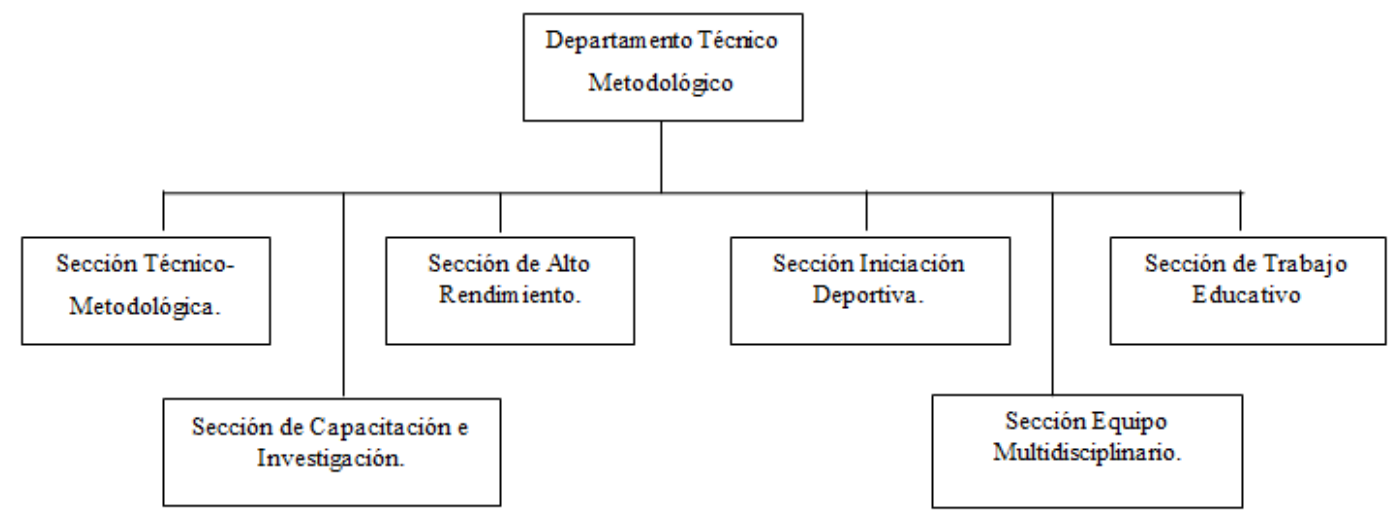

Fuente: El Autor (2013).

Con respecto al análisis y aprobación de los planes y programas de entrenamiento que constituye uno de los instrumentos más efectivos para conocer, analizar y evaluar la propuesta de trabajo del entrenador, se elaboró un protocolo de discusión con la finalidad que las propuestas sean valoradas cualitativa y cuantitativamente; Los aspectos que se controlaran en los Planes de Entrenamiento para mejorar los resultados deportivos serán: análisis del plan anterior, presentación del plan escrito y gráfico, estructura del plan gráfico, objetivos generales y específicos del macro-ciclo, períodos, etapas, test pedagógicos, competencias preparatorias y fundamentales, plan médico 
y psicológico, pronósticos y necesidades; La metodología a utilizar será; citar a todo el colectivo técnico para la discusión de su plan frente a los integrantes del Departamento Técnico Metodológico, con el objeto de realizar un trabajo integral que tendrá un tiempo de duración de 15 minutos para su exposición por parte del cuerpo técnico y 10 minutos para responder inquietudes del cuerpo multidisciplinario. Se calificará de acuerdo con la escala establecida para estos fines: Muy Buena (100 a 91), Buena; (90 a 71), Regular (70 a 61) y Mala (60 o menos).

Luego de revisar las visitas técnicas, metodológicas, a Ligas Deportivas Cantonales y a competencias que son aspectos muy importantes a tener en consideración al momento de realizar la planificación deportiva, porque de ésta deriva el control, seguimiento y evaluación del Departamento Técnico Metodológico hacia los estamentos subordinados, podemos sugerir que las: Visitas técnicas comprendan la revisión, evaluación y observación de la clase de entrenamiento en base al plan elaborado para el cuerpo técnico, en estas visitas, se debe planificar la presentación de la sesión de entrenamiento, control de la asistencia, información los objetivos de la clase, dirección del calentamiento, explicación de los ejercicios físicos, planificación de las pausas o descanso, corrección de errores, ejercicios de recuperación y evaluación final (Matveev,1983, pág. 96).

Tabla N. 1. Resultados de las visitas técnicas a las disciplinas deportivas de FEDECAÑAR (Federación Deportiva del Cañar).

\begin{tabular}{|c|c|c|c|c|c|c|c|c|c|}
\hline \multicolumn{10}{|c|}{ VISITAS TÉCNICAS } \\
\hline $\begin{array}{c}\mathbf{N} . \\
\mathbf{0}\end{array}$ & DEPORTE & $\begin{array}{c}\text { VISITA } \\
\mathbf{S}\end{array}$ & $\mathbf{1}$ & $\mathbf{2}$ & $\mathbf{3}$ & $\mathbf{4}$ & $\mathbf{5}$ & $\mathbf{6}$ & $\begin{array}{c}\text { EVALUACI } \\
\text { ÓN }\end{array}$ \\
\hline $\mathbf{1}$ & Ajedrez & 4 & 50 & 55 & 63 & 70 & & & $\mathrm{~B}$ \\
\hline $\mathbf{2}$ & Atletismo & 6 & 55 & 60 & 63 & 65 & 68 & 70 & $\mathrm{~B}$ \\
\hline $\mathbf{3}$ & Baloncesto & 2 & $\mathrm{X}$ & 63 & & & & & $\mathrm{R}$ \\
\hline $\mathbf{4}$ & Boxeo & 4 & 57 & 55 & 58 & 62 & & & $\mathrm{R}$ \\
\hline
\end{tabular}




\begin{tabular}{|c|c|c|c|c|c|c|c|c|c|}
\hline $\mathbf{5}$ & Escalada & 5 & 53 & 58 & 62 & 65 & 70 & & $\mathrm{~B}$ \\
\hline $\mathbf{6}$ & Futbol & 2 & 50 & 75 & & & & & $\mathrm{~B}$ \\
\hline $\mathbf{7}$ & $\begin{array}{c}\text { Fis. } \\
\text { Culturismo }\end{array}$ & $\mathrm{X}$ & $\mathrm{X}$ & & & & & & $\mathrm{X}$ \\
\hline $\mathbf{8}$ & Gimnasia "D" & 2 & 56 & 77 & & & & & $\mathrm{~B}$ \\
\hline $\mathbf{9}$ & Gimnasia "V" & 2 & 51 & 69 & & & & & $\mathrm{R}$ \\
\hline $\mathbf{1 0}$ & Judo & 6 & 80 & 84 & 88 & 90 & 90 & 91 & $\mathrm{EX}$ \\
\hline $\mathbf{1 1}$ & Karate Do & 4 & 68 & 75 & 78 & 80 & & & $\mathrm{MB}$ \\
\hline $\mathbf{1 2}$ & Lev. Pesas & 4 & 79 & 74 & 77 & 79 & & & $\mathrm{~B}$ \\
\hline $\mathbf{1 3}$ & Lucha & 6 & 85 & 88 & 89 & 90 & 88 & 90 & $\mathrm{EX}$ \\
\hline $\mathbf{1 4}$ & Tae Kwon Do & 4 & 75 & 78 & 80 & 84 & & & $\mathrm{MB}$ \\
\hline $\mathbf{1 5}$ & Patinaje & 3 & 58 & 63 & 75 & & & & $\mathrm{~B}$ \\
\hline \multicolumn{7}{|l}{ Total de Visitas } & $\mathbf{5 4}$ & &
\end{tabular}

Fuente: El Autor (2013).

Si observamos en la tabla 1 se plantearon 54 visitas técnicas, que se distribuyeron en las 14 de las 15 disciplinas deportivas de las cuales se obtuvo lo siguiente: 2 disciplinas tienen la calificación de Excelente, 2 con calificación Muy Buena, 7 con calificación Buena, 3 con calificación Regular, 1 deporte que no fue considerado para las visitas como lo indica el cuadro.

Se puede evidenciar que en la mayoría de disciplinas deportivas conoce muy poco sobre la ayuda que brindan la Planificación del Entrenamiento Deportivo en el desarrollo físico y técnico del deportista, demostrando así que el cuerpo técnico no está considerando las estrategias y peor aún las técnicas metodológicas físico-deportivas aplicables en la actualidad, mismas que tomando en consideración van a ayudar al desarrollo y al mejoramiento del rendimiento deportivo de sus dirigidos. Además, debo indicar que es imperioso que los integrantes del cuerpo técnico deben estar siempre prestos a las innovaciones técnicas, con el mayor objetivo de ofrecer mejores condiciones a los deportistas que practican tal o cual deporte. 
Gráfico N. 1. Resultados de las Visitas Técnicas realizadas a las Disciplinas Deportivas de FEDECAÑAR (Federación Deportiva del Cañar).

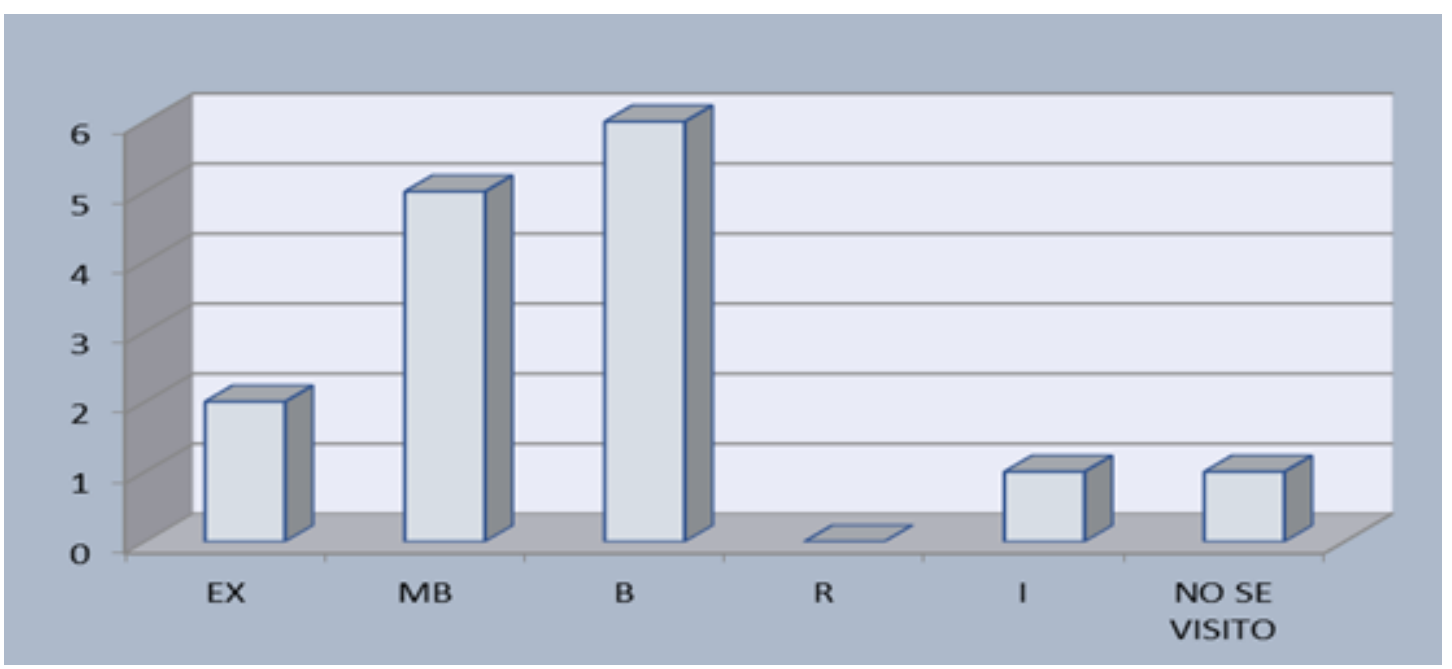

Fuente: El Autor (2013).

Las visitas metodológicas deben contemplar: 1. El chequeo de la documentación del plan de entrenamiento que va dirigido a los deportistas que tienen un proceso de entrenamiento o programa de enseñanza que direcciona la preparación de los deportistas de iniciación; 2. La impartición de disposiciones que deben ser direccionadas a los técnicos. Se debe evaluar la presentación del plan escrito, gráfico, unidades de entrenamiento, análisis del mesociclo, microciclo, control de Test Pedagógicos, análisis de resultados, registro de asistencia, calendario competitivo y su pronóstico.

Tabla N. 2. Resultados de las visitas metodológicas realizadas a las disciplinas deportivas de FEDECAÑAR (Federación Deportiva del Cañar).

\begin{tabular}{|c|c|c|c|c|c|c|c|c|c|}
\hline \multicolumn{10}{|c|}{ VISITAS METOdOLÓGICAS } \\
\hline $\begin{array}{c}\text { N. } \\
0\end{array}$ & DEPORTE & $\begin{array}{c}\text { VISITA } \\
\text { S }\end{array}$ & $\mathbf{1}$ & $\mathbf{2}$ & $\mathbf{3}$ & $\mathbf{4}$ & $\mathbf{5}$ & $\mathbf{6}$ & $\begin{array}{c}\text { EVALUACI } \\
\text { ÓN }\end{array}$ \\
\hline $\mathbf{1}$ & Ajedrez & 4 & 70 & 79 & 84 & 89 & & & MB \\
\hline
\end{tabular}




\begin{tabular}{|c|c|c|c|c|c|c|c|c|c|}
\hline $\mathbf{2}$ & Atletismo & 6 & 70 & 70 & 73 & 79 & 81 & 83 & $\mathrm{MB}$ \\
\hline $\mathbf{3}$ & Baloncesto & 2 & 65 & 79 & & & & & $\mathrm{~B}$ \\
\hline $\mathbf{4}$ & Boxeo & 4 & 60 & 61 & 65 & 70 & & & $\mathrm{~B}$ \\
\hline $\mathbf{5}$ & Escalada & 5 & 60 & 60 & 65 & 70 & & & $\mathrm{~B}$ \\
\hline $\mathbf{6}$ & Fútbol & 2 & 68 & 77 & & & & & $\mathrm{~B}$ \\
\hline $\mathbf{7}$ & $\begin{array}{c}\text { Fis. } \\
\text { Culturismo }\end{array}$ & $\mathrm{X}$ & $\mathrm{X}$ & & & & & & $\mathrm{X}$ \\
\hline $\mathbf{8}$ & Gimnasia & 2 & 64 & 79 & & & & & $\mathrm{~B}$ \\
\hline $\mathbf{9}$ & $\begin{array}{c}\text { Gimnasia } \\
\text { "V" }\end{array}$ & 2 & 66 & 80 & & & & & $\mathrm{I}$ \\
\hline $\mathbf{1 0}$ & Judo & 6 & 84 & 86 & 88 & 88 & 90 & 90 & $\mathrm{EX}$ \\
\hline $\mathbf{1 1}$ & Karate Do & 4 & 79 & 70 & 77 & 78 & & & $\mathrm{~B}$ \\
\hline $\mathbf{1 2}$ & Lev. Pesas & 4 & 80 & 81 & 83 & 83 & & & $\mathrm{MB}$ \\
\hline $\mathbf{1 3}$ & Lucha & 6 & 87 & 89 & 88 & 90 & 91 & 93 & $\mathrm{EX}$ \\
\hline $\mathbf{1 4}$ & $\begin{array}{c}\text { Tae Kwon } \\
\text { Do }\end{array}$ & 4 & 78 & 80 & 81 & 87 & & & $\mathrm{MB}$ \\
\hline $\mathbf{1 5}$ & Patinaje & 3 & 70 & 79 & 84 & & & & $\mathrm{MB}$ \\
\hline \multicolumn{2}{|l|}{ Total de Visitas } & $\mathbf{5 4}$ & \multicolumn{7}{|l}{} \\
\hline
\end{tabular}

Fuente: El Autor (2013).

Se plantearon 54 visitas técnicas, distribuidas en las 14 de las 15 disciplinas deportivas de las cuales se obtuvo: 2 tiene la calificación de Excelente, 2 de calificación Muy Buena, 7 con calificación Buena, 3 con calificación Regular, 1 deporte que no fue considerado para las visitas como lo indica el cuadro.

Con relación al ítem planteado se logró determinar que el cuerpo técnico encuestado desconoce sobre los beneficios de tener los documentos como el plan escrito, gráfico, unidades de entrenamiento, análisis del mesociclo, microciclo, control de Test Pedagógicos, análisis de resultados, registro de asistencia, calendario competitivo y su pronóstico, como medio de ayuda en el proceso enseñanza-aprendizaje de sus dirigidos. 
Gráfico N.․ 2. Resultados de las Visitas Metodológicas realizadas a las

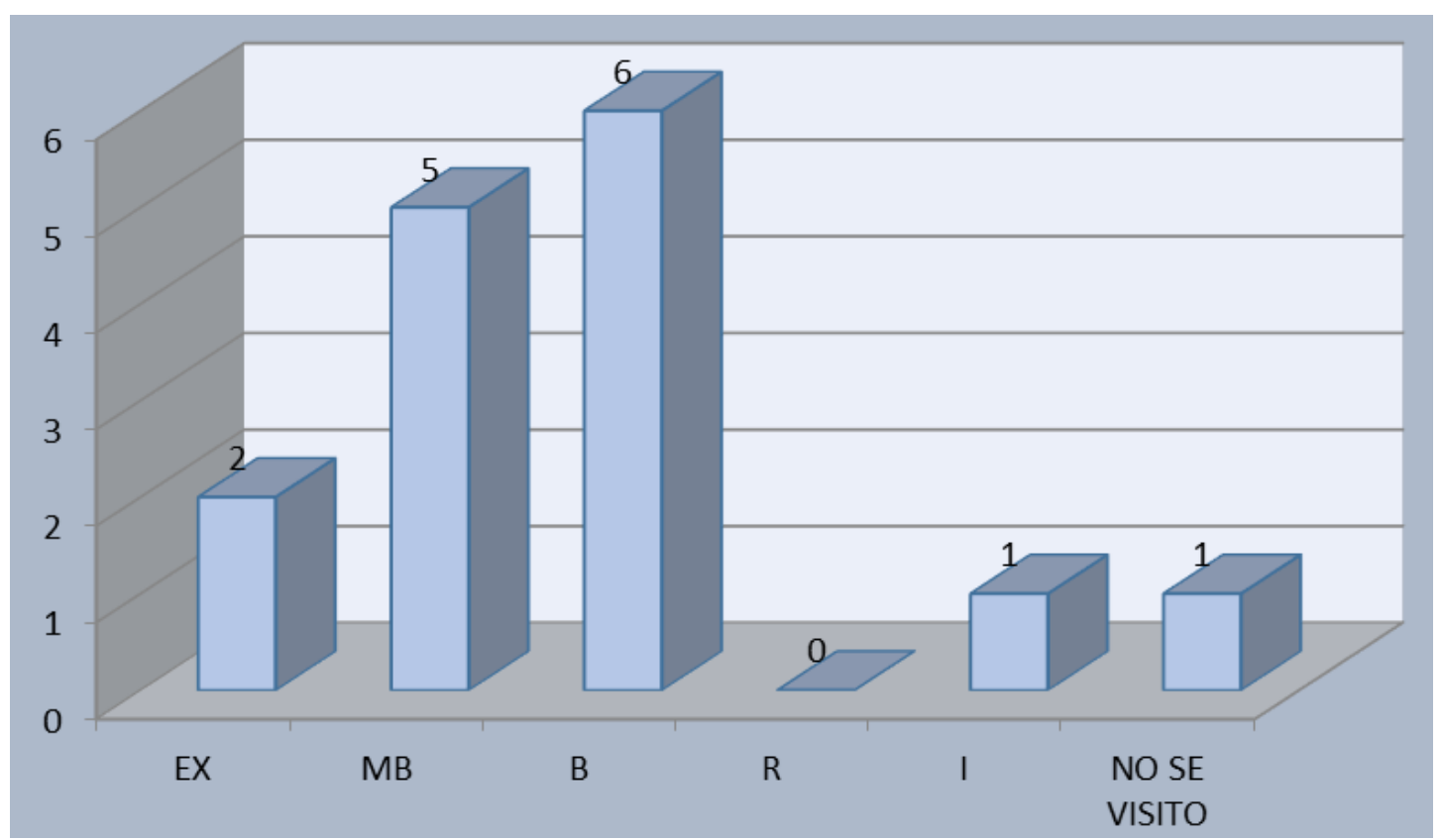

Fuente: El Autor (2013).

En las visitas a Ligas Deportivas Cantonales se controlará el Plan anual y trimestral de actividades, inventario de las instalaciones deportivas, característica de la fuerza técnica, y el plan de capacitación. Las visitas a competencias, en este tipo de visitas se controlarán si existe un comité de recepción, el congresillo técnico, escenario deportivo, implementación, organización, calendario de competencias, seguridad, Juzgamiento etc.

En cuanto a las reuniones con el cuerpo técnico cumplirán objetivos fundados de la revisión de los problemas que provienen del trabajo técnico metodológico, lo que permitirá dar solución de primera mano a los problemas antes que afecten la preparación de los deportistas.

En el caso de nuestra provincia, el problema de la conformación ha sido inmenso por el desconocimiento de la dirigencia del trabajo que debe cumplir el equipo multidisciplinario (Médico/a, Psicólogo/a, Fisioterapeuta, 
Trabajador/a Social) en el proceso de entrenamiento deportivo, para realizar un trabajo adecuado y acorde a los objetivos establecidos por la institución. Este equipo de profesionales, coadyuvarán a mejorar el proceso del entrenamiento deportivo, manteniendo al deportista en un nivel de trabajo adecuado, en lo biopsicosocial y ecológico y tendrá relación directa con el Departamento Técnico Metodológico.

En el aspecto de la evaluación a la fuerza técnica deberá estar establecida por una normativa de acuerdo con la ley de Educación Física Deportes y Recreación que rige en nuestro país y el estatuto vigente de la Federación Deportiva del Cañar, por lo que se plantea los siguientes objetivos:

1. Lograr que el entrenador ejecute un mejor trabajo, tomando en cuenta los parámetros establecidos en la escala de puntuación, lo que asegurará su estabilidad laboral.

2. Mantener un control apropiado del trabajo de todos los miembros de la fuerza técnica de la Federación Deportiva Provincial de Cañar de acuerdo con los objetivos propuestos para el año, entre los aspectos a controlar está: cumplimiento del plan de entrenamiento, resultados competitivos, aporte de deportistas a selecciones nacionales, superación profesional y el nivel de coordinación con el cuerpo multidisciplinario.

Sobre los resultados obtenidos en los aspectos antes mencionados, se considera de mucha importancia la implementación de un plan de capacitación y masificación provincial, documento que debe ser estructurado de acuerdo con la realidad de Federación Deportiva Provincial del Cañar; y, para ello, se planteó varias estrategias, entre las que consta, la realización de una encuesta a todo el personal deportivo, consultando temas generales y específicos, con el propósito de que sean los técnicos y monitores los que determinen, los 
temas de interés vinculados a su campo de acción.

\section{Recomendación}

Por todas las conclusiones antes expuestas, se recomienda que la propuesta planteada sea ejecutada dentro del Departamento Técnico Metodológico de la Federación Deportiva del Cañar; expuesta a las federaciones provinciales y a las universidades que cuenten con la carrera de Cultura Física, con el objeto de que sea considerada dentro de la malla curricular.

\section{Conclusiones}

Luego del trabajo de investigación realizado y por los resultados obtenidos a través del F.O.D.A. llegamos a las siguientes conclusiones, que avalan la propuesta presentada:

1. La estructura orgánica del Departamento Técnico Metodológico de la Federación Deportiva del Cañar no posee secciones de dirección metodológico lo que no le permite atender las necesidades reales de la provincia.

2. La aprobación de los planes de entrenamiento realizados al cuerpo técnico, se lo hace de manera superficial y sin analizar los planes anteriores. A demás no se cuenta con la presencia del cuerpo multidisciplinario produciendo bajo rendimiento deportivo.

3. Las visitas realizadas a los diferentes estamentos no cuentan con lineamientos claros para su ejecución.

4. No se mantienen reuniones periódicas con los técnicos, lo que impide atender las necesidades presentes en el proceso de entrenamiento.

5. La dirigencia deportiva desconoce la importancia de contar con un cuerpo multidisciplinario en el entrenamiento deportivo de los atletas, con el objeto de mejorar el rendimiento deportivo y de formar seres 
humanos integrales.

6. No se cuenta con un plan establecido para masificar el deporte, ni para capacitar al cuerpo técnico, acorde a las necesidades del tipo de deporte y las características de los atletas.

\section{Referencias}

Forteza, A., \& Ranzola, A. (1988). Bases Metodológicas del Entrenamiento Deportivo. La Habana, Cuba: Editorial Científico-técnica, págs. 84.

Matveev, L. (1983). Fundamentos del entrenamiento deportivo. Moscú, Rusia: Editorial Raduga.

Montero, R. (2006). Apuntes sobre los procesos y condicionantes. España: Paidotribo.

Vinueza, E, (2004a,b,c,d). Estructuración y Organización de los Departamentos Técnicos Metodológicos Nacionales y Provinciales. Tena, Ecuador: Federación Deportiva Provincial De Napo. Inédito.

Vasconcelos, A. (2000). Planificación y Organización de Entrenamiento Deportivo. Barcelona, España: Editorial Paidotribo. 


\section{Olger Estuardo Almache Tello}

e-mail: estuardo.almache@unae.edu.ec

Nacido en Azogues, Ecuador. Máster en Cultura Física

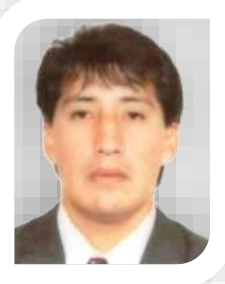

por la Universidad de Guayaquil. Catedrático de deportes de la Universidad Nacional de Educación. Catedrático de Cultura Física de la Universidad Católica de Cuenca sede Azogues. Director del Departamento Técnico Metodológico de la Federación Deportiva del Cañar desde 2001 hasta el 2014. Docente de Cultura Física de la Escuela Gabriela Mistral desde 1998 hasta el 2011. Docente de Educación Física del Centro Educativo la Esperanza desde 1997 hasta el 2001. 